DOI: https://dx.doi.org/10.26808/rs.ca.i8v4.04 International Journal of Computer Application (2250-1797)

Issue 8 Volume 4, July-August 2018

\title{
Coverage probability of HetNet using Stochastic geometry and Poisson Point Process
}

\author{
Gitimayee Sahu \\ Student Member IEEE,Dept. of Electronics and Telecommunication \\ UMIT, Juhu, Mumbai \\ Sanjay S. Pawar \\ Senior Member IEEE \\ Dept. of Electronics and Telecommunication, UMIT, Juhu, Mumbai, India
}

\begin{abstract}
The exponential growth in the number of cellular users along with their increasing demand of higher transmission rate, ultra low latency, guaranteed QoS and lower power consumption is of prime importance for the design of future generation networks. The spectral efficiency (SE) can be improved by better utilization of the network resources at the cost of reduction in the energy efficiency (EE) due to increase in the network power expenditure arising from densification of the network. One of the possible solution is to deploy Heterogeneous Network (HetNet) consisting of several tiers of small cell BSs overlaid within the coverage area of the macrocell. The HetNet can provide better coverage and data rate to the cell edge users in comparison to the macrocell only deployment. Therefore, optimal network planning is an important challenge for future heterogeneous network with high number of small cells. In order to tackle these challenges, here we focus on the random spatial modeling of Multi Tier Heterogeneous Network consisting of dense small cell access points using Poisson Point Process (PPP) with Vornoi Tessellation. Then we calculate the coverage and outage probability of the cellular network by using stochastic geometry.
\end{abstract}

Index Terms-Heterogeneous network; Poisson point process; coverage probability

\section{INTRODUCTION}

According to the recent reports from Cisco [1], the monthly global mobile data traffic is expected to reach 24.3 Exabyte by end of 2019, wherein 75 per cent of the total monthly mobile traffic will be video. Almost 80 per cent of the total video based on monthly data traffic will originate from indoor. The traditional cellular deployment consisting of macrocell cannot cope with this ever increasing data traffic demands. Dense deployment of macrocells in order to enhance the coverage area and increased number of mobile subscribers are not feasible due to their high deployment cost [2], [3]. Due to this fact, a paradigm shift is required for the next generation communication network. One of the emerging paradigm proposed for the next generation communication network is the fifth Generation $(5 \mathrm{G})$ network to provide 1000 times more capacity along with the data rates in the range of 1 Giga bits per second (Gbps), end-to-end delay of 1 milli second(ms) and 100 times less energy consumption in comparison to the current cellular network[4]. The promising nine enabling technologies for $5 \mathrm{G}$ network are identified as [3].

i. Heterogeneous network (HetNet), ii. Device-to-device (D2D) communication, iii. Massive multiple-input multiple-output (MIMO), iv. Millimeter wave (mmWave), v. Full duplex 
DOI: https://dx.doi.org/10.26808/rs.ca.i8v4.04 International Journal of Computer Application (2250-1797)

Issue 8 Volume 4, July-August 2018

communication, vi. Energy-aware communication, vii. Energy harvesting, viii. Cloud-based radio access network(C-RAN) and ix. Virtualisation of network resources. Among these, one of the promising solutions is HetNet, which provides better coverage at the cell edge and higher data rate. The HetNet include low-power overlaid BSs (or small cells), e.g., microcell, picocell and femtocell, within the macrocell geographical area, deployed by either the user or the network operator who share the same spectrum with the macrocell [4]. The purpose of HetNet is to allow the user equipment (UE) to access the small cells overlap geographical coverage areas even though the UEs are within the macrocell [5]. The deployment of small cells has a great potential to improve the spatial reuse of radio resources and also to enhance the transmit power efficiency [6], and in turn, the network EE.

Device-to-Device (D2D) communication is an additional promising technique which can be integrated by cellular network providers to fulfill the spectral and energy efficiency requirement for the future $5 \mathrm{G}$ wireless network [7]. D2D communication can significantly improve the resource utilization due to the hop gain, the proximity gain and the reuse gain. Each promising solution alone is unlikely to meet the QoS and throughput requirement for 5G [2]. One of the promising solutions is a hierarchical HetNet in which the above mentioned technologies such as HetNet and D2D can coexist in parallel to improve the network performance.

EE is, in fact, one of the key performance indicators for the next generation wireless communication system. The motivation behind the EE arises due to the current energy cost payable by operators for running their access network as a significant factor of their operational expenditures (OPEX). Hence, green networking paradigm, which focuses on the means to reduce the energy consumption in the wireless access networks, has received a lot of attention [8]. One of the fundamental system design requirement for the future generation, such as the Fifth generation $(5 \mathrm{G})$ network is to jointly optimize contradicting objectives, e.g., to provide reliable coverage with higher SE and lower energy consumption and lower cost per information transfer [9].

\section{Literature Survey}

$5 \mathrm{G}$ will need to be a paradigm shift that includes very high carrier frequencies with massive bandwidths, extreme base station and device densities, and unprecedented numbers of antennas. However, unlike the previous four generations, it will also be highly integrative: tying any new 5G air interface and spectrum together with LTE and Wi-Fi to provide universal high-rate coverage and a seamless user experience [2-4].

In 5G the challenges like X1000 times capacity, X10 to 100 times data rate can be addressed by bringing the end points closer together, i.e. for indoor users $(>70 \%)$ can be provided with indoor solutions e.g. small cell [13]. The technical, research, infrastructure, business and standardization aspects of femto cell are addressed in the literature [8],[29]. The small cell will make the cellular network heterogeneous (HetNet). Modeling of the k-tier HetNet can be done using Poisson point process (PPP) random spatial modeling [17-18], [22], [24], [27-29]. The coverage probability can be improved and the outage probability can be reduced by using HetNet [34-37].

As femto cells have self organizing, self healing and self optimizing capability hence deployment of small cell can be adaptive [16],[30],[32]. Efficient resource allocation of the small cell can be done by dedicatedly assigning some of the resources of the macro cell to the small cell and using Absolute blank sub frame(ABS) [7],[9],[16],[26]. The dense deployment of small cell arise inter channel (small cell to small cell) and intra channel (macro cell to small cell) interference. This can be minimized by cognitive theory and by adopting power control strategy [5],[10],[19], 20]. The spectrum efficiency (SE) of ultra dense network can 
be enhanced by using co-operative bargaining game theory. The energy efficiency (EE) can be improved by sleep mode mechanism (i.e. green communication technique) when the traffic level is below threshold [84], [47]. When the macro BS is congested, then by mobile traffic offloading to femto cell either by co-operative game theory [46] or by markov`s queuing theory [82] the improvement of EE of the Macro BS and SE of the small BS can be obtained [48],[49-53]. By using Nash equilibrium game theory a trade-off relationship between the SE and EE efficiency can be established [44],[45].

Different types of path loss mechanisms like Rayleigh fading, Rician fading, Okumara hata, Friis transmission equation, shadowing, fading and lognormal shadowing[64][71]. Regarding wireless backhaul network for dense small cell network, centralized and distributed scenario are efficiently used [54]-[60]. The mobile femtocell can be widely used for mobile users using cloud RAN(C-RAN) concept [61]-[63].

Different types of game theory techniques, for example, resource allocation for wireless co-operative network [72], dynamic bargaining for relay based co-operative spectrum sharing [73], power allocation and pricing in multiuser relay networks using stackelberg and bargaining games [74], spectrum leasing as an incentive framework towards uplink of macrocell and femtocell co-operation [75], Spectrum leasing to co-operative secondary ad-hoc network [76], Spectrum mobility game [77] local base station co-operation via finite-capacity links for the uplink of linear cellular networks [78]. By using cognitive concept, sharing and sensing the frequency carrier and by using power control mechanism the spectral density of the ultra dense cellular network can be enhanced [79]-[83]. By using the fractional frequency reuse (FFR) concept inter tier and intra tier interference can be minimized [84].

\section{Problem Statement}

To investigate the coverage probability of spatial random model of BS and to compare it with the regular grid model of wireless cellular network. Also investigate the effect of coverage probability in a two tier cellular network by increasing the number of macro BS and by increasing the density of Small BS.

\section{System Model}

Consider a cellular network with $\mathrm{Nb}$ BSs transmitting on the same time frequency resources. Consider a randomly selected user is served by a BS located at a distance $R_{0}$. The selection of the serving BS is based on a predefined selection law, e.g., based on the maximum received power. All the other (Nb-1) BSs, except the serving BS, act as interferers. Denoting the distance of the randomly selected user to these interferers by $\left\{R_{i}\right\}$ where the subscript $1 \leq i<N_{b}$ denotes the index of the interferer. All the transmitters are assumed to transmit at a fixed power P. Each wireless link suffers from a distance based on path-loss with exponent $\alpha$ and Rayleigh fading. Therefore, the received power at a distance $r$ from the transmitter is $P_{r}=P h r^{-\alpha}$. Where h exp(1) models Rayleigh

fading. The thermal noise is assumed to be N. The downlink SINR for the randomly chosen user in this setup i.e. fig. 1 is given by,

$$
S I N R=\frac{P h R_{0}^{-\alpha}}{\sum_{i=1}^{N_{b}-1} P h_{i} R_{i}^{-\alpha}+N} .
$$

By neglecting the thermal noise N, the SINR will be termed as SIR. The SIR is a random variable due both to the random channel gains and the random distances to the serving $\mathrm{BS}$ 
DOI: https://dx.doi.org/10.26808/rs.ca.i8v4.04 International Journal of Computer Application (2250-1797)

Issue 8 Volume 4, July-August 2018

and the interferers. The coverage probability $(\mathrm{Pc})$ for this general setup can be derived as follows:

$$
P c=P(\operatorname{SIR}>\gamma)
$$

We first specialize the analysis to the hexagonal grid model i.e. fig.2 in the following subsection.

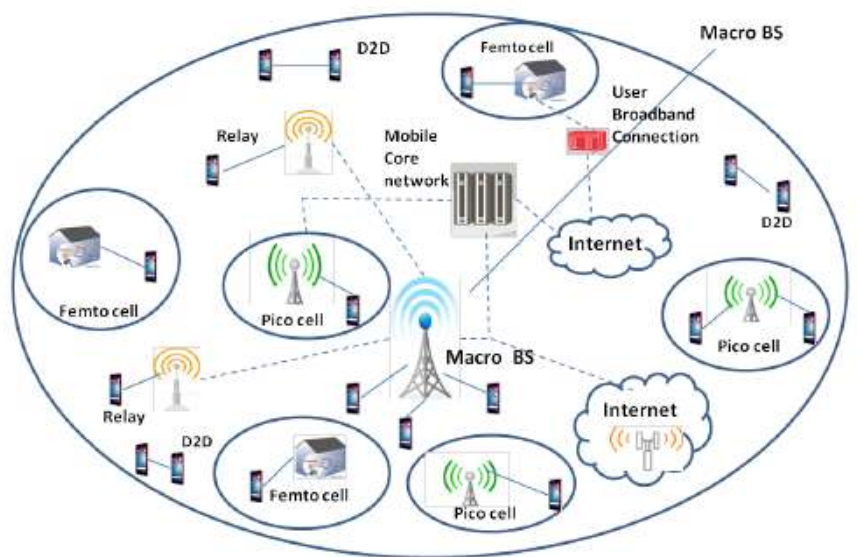

Fig. 1: Heterogeneous cellular wireless network Model

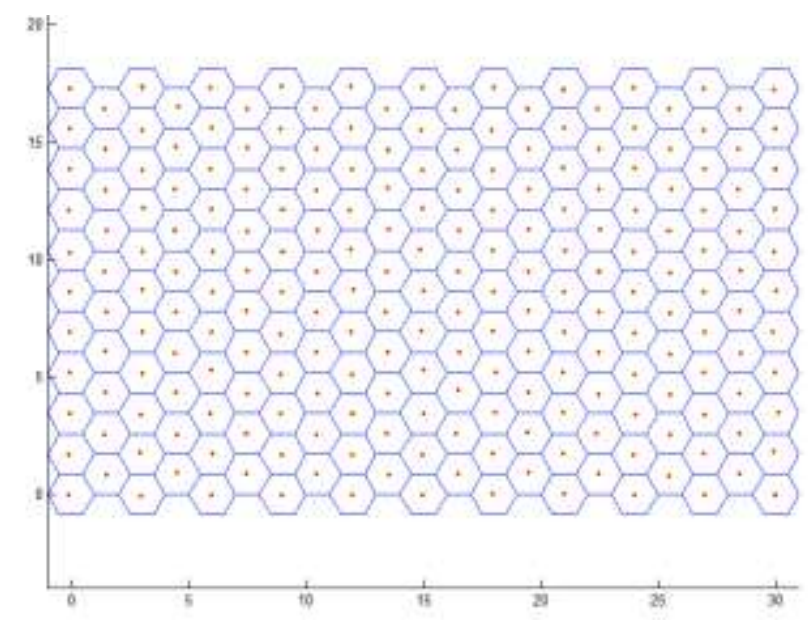

Fig. 2: Seven-cell hexagonal grid model with BSs denoted by red dots.

\section{A. Coverage Probability using Hexagonal Grid Model}

Consider a seven-cell version of the grid model shown in Fig. 2, where the interference field is limited to the first ring of interferers. Note that limiting the interference field to first or at most second ring of interferers is a common practice in literature [8]. The inter-site distance is assumed to be $D$. We assume maximum average power based cell selection, where each user lying in a particular hexagon is served by the BS lying at the center of that hexagon. For downlink analysis, a user uniformly distributed in the center cell is selected. In addition to its distance to the serving BS, its location is characterized by the angle $\theta$ it makes with the horizontal axis, with the origin being the BS corresponding to the center cell. For this setup, the distance of the chosen user to the $i_{t h}$ interferer can be expressed as follows:

$$
R_{i}=\sqrt{\left(D \cos \theta_{i}-R_{0} \cos \theta\right)^{2}+\left(D \sin \theta_{i}-R_{0} \sin \theta\right)^{2}} \text {. }
$$




$$
P c=P(\operatorname{SIR}>\beta)
$$

With $\theta_{i}$ being the angle formed by the location of the $i_{t h}$ interfere with horizontal axis. The values of $\theta_{i}$ for all the six interferers are as follows : $\theta_{1}=\frac{\pi}{6}, \theta_{2}=\frac{\pi}{2}, \theta_{3}=\frac{5 \pi}{6}, \theta_{4}=\frac{7 \pi}{6}, \theta_{5}=\frac{3 \pi}{2}$ and $\theta_{6}=\frac{11 \pi}{6}$.

$R_{i}$ is random because of its dependence on $R_{0}$ and $\theta$. The general coverage probability expression given by (4) can be specialized for this case to

$$
P_{C}=E_{R_{0}, \theta}\left[\prod_{i=1}^{6} \frac{1}{1+\beta R_{0}^{\alpha}\left(\left(D \cos \theta_{i}-R_{0} \cos \theta\right)^{2}+\left(D \sin \theta_{i}-R_{0} \sin \theta\right)^{2}\right)^{-\frac{\alpha}{2}}}\right] \text { (5) }
$$

Where, the expectation is with respect to the joint distribution of $R_{0}$ and $\theta$.

\section{B. Coverage Probability using Random Spatial Model}

Assume that the BS locations are modeled by a homogeneous PPP $\phi$ with density $\lambda$, as shown in Figure 3. The users are also modeled by an independent PPP. Without loss of generality, the downlink analysis is conducted at the typical mobile user located at the origin. Assume that the user connects to the BS that provides the maximum average received power, i.e. the one that is closest to the origin. Using null probability of a PPP, the distribution of the distance of the typical user to the serving BS is

$$
f_{r_{0}}\left(r_{0}\right)=2 \pi \lambda r_{0} \exp \left(-\lambda \pi r_{0}^{2}\right) \text {. }
$$

The coverage probability expression can be simplified using simple tools from stochastic geometry as follows:

$$
\begin{aligned}
& P_{c}=\int_{0}^{\infty} E_{\phi}\left[\sum_{\substack{x_{i} \in \phi \\
\left\|x_{i}\right\|>r}} \frac{1}{1+\beta r^{\alpha}\left\|x_{i}\right\|^{-\alpha}}\right] f_{R_{0}}(r) d r . \\
& P_{c}=\int_{0}^{\infty} \exp \left(-2 \pi \lambda \int_{0}^{\infty} 1-\frac{1}{1+\beta r^{\alpha} u^{-\alpha}} u d u\right) f_{R_{0}}(r) d r . . \\
& P_{c}=\int_{0}^{\infty}\left(-\pi \lambda r^{2} \beta^{2 / \alpha} \int_{\beta-\frac{2}{\alpha}}^{\infty} \frac{1}{1+v^{\alpha / 2}} d v\right) f_{R_{0}}(r) d r \\
& P_{c}=\int_{0}^{\infty} \exp \left(-\pi \lambda r^{2} \rho(\alpha, \beta)\right) f_{R_{0}}(r) d r \text {. } \\
& P_{c}=\frac{1}{1+\rho(\alpha, \beta)}
\end{aligned}
$$

Where equn.8 follows from the probability generating functional (PGFL) of a PPP, equn.9 follows by the substitution $\beta^{-\frac{2}{\alpha}} r^{-2} u^{2} \rightarrow v$. In equn. 10 the constant is defined by,

$$
\rho(\alpha, \beta)=\beta^{2 / \alpha} \int_{\beta-\frac{2}{\alpha}}^{\infty} \frac{1}{1+v^{\alpha / 2}} d v
$$

Equn. 11 follows by substituting the distribution of R0. Here the coverage probability reduces to a closed form expression. These expressions are useful to gain key system design insights. From the above expression it is clearly shown that the coverage probability is independent of the density of the BSs when the cellular network is interference limited. 


\section{System Model of 2-tier HetNets}

Here we model a 2-tier HetNet as a cellular network where each tier model the BSs of a particular class, such as those of femtocells or pico-cells. The BSs across tiers may differ in terms of the transmit power, the supported data rate and their spatial density. We assume that the BSs in the ith tier are spatially distributed as a PPP $\phi_{i}$ of density $\lambda_{i}$ transmit at power Pi and have a SINR target of $\beta_{i}$. More precisely a mobile can reliably communicate with an $i^{\text {th }}$ tier BS located at $x_{i}$ belongs to $\phi_{i}$ only if its downlink SINR with respect to that BS is greater than $\beta_{i}$. Thus, each tier can be uniquery defined by the tuple $P_{i}, \beta_{i}, \lambda_{i}$. Without loss of generality, the analysis is conducted on a typical mobile user which is located at the origin. The fading (power) between a BS located at point $\mathrm{x}$ and the typical mobile is denoted by $h_{x}$ and is assumed to be i.i.d exponential (Rayleigh fading). The standard path loss function is given by $l(x)=\|x\|^{-\alpha}$, where $\alpha>2$ is the path loss exponent. Hence, the received power at a typical mobile user from a BS located at point $x_{i}$ belongs to $\phi_{i}$ is $P_{i} h_{x i}\left\|x_{i}\right\|^{-\alpha}$ where $h_{x i} \sim \exp (1)$. The resulting SINR expression assuming the user connects to this BS is given by

$$
\operatorname{SINR}\left(x_{i}\right)=\frac{P_{i} h_{x i}\left\|x_{i}\right\|^{-\alpha}}{\sum_{j=1}^{k} \sum_{x \in \phi_{j}} P_{j} h_{x}\|x\|^{-\alpha}}
$$

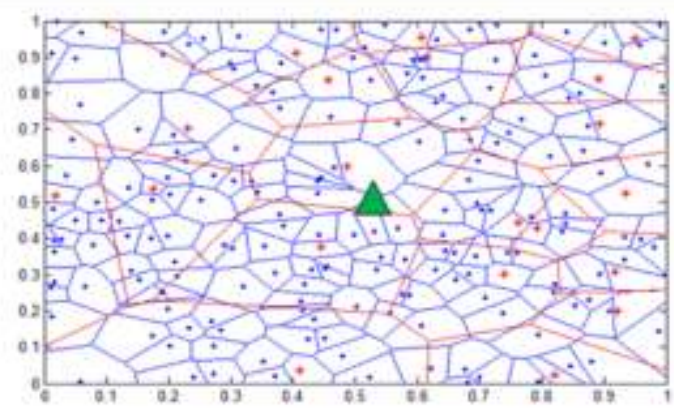

Figure 3: Voronoi tessellation of the macro BSs PPP, shown in red stars, and the SCs PPP denoted by the blue dots. The UE under study is located in the centre of the network denoted by the green triangle

\section{Coverage Probability}

A typical mobile user is said to be in coverage if it is able to connect to at least one BS with SINR above its threshold. In this case when all the tiers have same SINR threshold $\beta$, coverage probability is the complementary cumulative distribution function (CCDF) of the effective received SINR, outage being the CDF, i.e., $1-C C D F$. In an interference limited network, i.e., when self-interference dominates thermal noise, the coverage probability of a typical mobile user simplifies to,

$$
P_{C}\left(\lambda_{i}, \beta_{i}, P_{i}\right)=\frac{\pi}{C(\alpha)} \frac{\sum_{i=1}^{k} \lambda_{i} P_{i}^{\frac{2}{\alpha}} \beta_{i}^{-2 / \alpha}}{\sum_{i=1}^{k} \lambda_{i} P_{i}^{\frac{2}{\alpha}}} . .
$$


DOI: https://dx.doi.org/10.26808/rs.ca.i8v4.04 International Journal of Computer Application (2250-1797)

Issue 8 Volume 4, July-August 2018

\section{The Potential Throughput}

The potential throughput can directly obtained from the coverage probability expressions. The potential throughput is given by:

$$
R(\lambda, \theta)=\lambda \exp \left(-\frac{\pi \lambda}{\alpha} \ln (1+\theta)\right) \log _{2}(1+\theta) . .(15)
$$

Based on the above expression the potential throughput approaches zero when $\lambda \rightarrow \infty$

\section{Simulation Setup}

The network under simulation consists of two coexisting realizations of homogenous and independent PPPs which represent the Macro BS and SC tiers with intensity $\lambda_{M B S}$ and $\lambda_{S B S}$. From the fig. 4 by introducing more cells into the network, the coverage would increase. However, as the number of macro BSs increases, the coverage probability tends to saturate to a maximum value. This is due to the fact that increasing the number of cells increases at the same time interference at the UE. In the fig. 4 the coverage probability for a single tier small cell network is presented. Here we plot the probability of coverage versus the SC intensity $\lambda_{S C}$. In the fig. 10 the probability of coverage vs. the small cell intensity in a two tier network for different values of macro BS(i.e. one to four). The probability of coverage is increasing by adding more macro BSs into the HetNet. Again by increasing the number of macro BSs results in gradually decreasing the slope of the coverage probability due to interference raise.

Table 1: Simulation parameters

\begin{tabular}{|c|c|c|c|}
\hline S No. & Simulation Parameter & Symbol & Value \\
\hline 1. & BS Transmission Power & $P_{B S}$ & $48 d B m$ \\
\hline 2. & SC Transmission Power & $P_{S} C$ & $12 d B m$ \\
\hline 3. & Pathloss Exponent & $\alpha$ & 4 \\
\hline 4. & Rayleigh fading channel ooef. (mean) & $\mu$ & 1 \\
\hline 5. & Thermal Noise Power & $N$ & $-100 d B m$ \\
\hline 6. & Simulation area & $A$ & $1 \mathrm{~km}^{2}$ \\
\hline
\end{tabular}

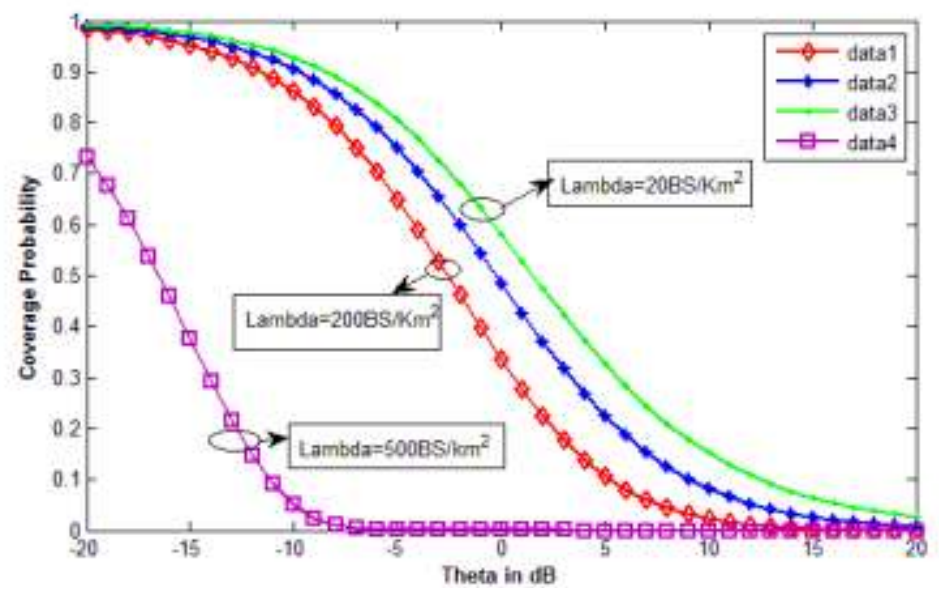

Figure 4: The coverage probability vs. $\theta$ for different BS densities assuming different path loss models 
DOI: https://dx.doi.org/10.26808/rs.ca.i8v4.04 International Journal of Computer Application (2250-1797)

Issue 8 Volume 4, July-August 2018

Setting $\mathrm{K}=1$ leads to the single-tier case, where the coverage probability is given by:

$P_{c}(\lambda, \beta, P)=\frac{\pi}{C(\alpha) \beta^{2 / \alpha}}$.

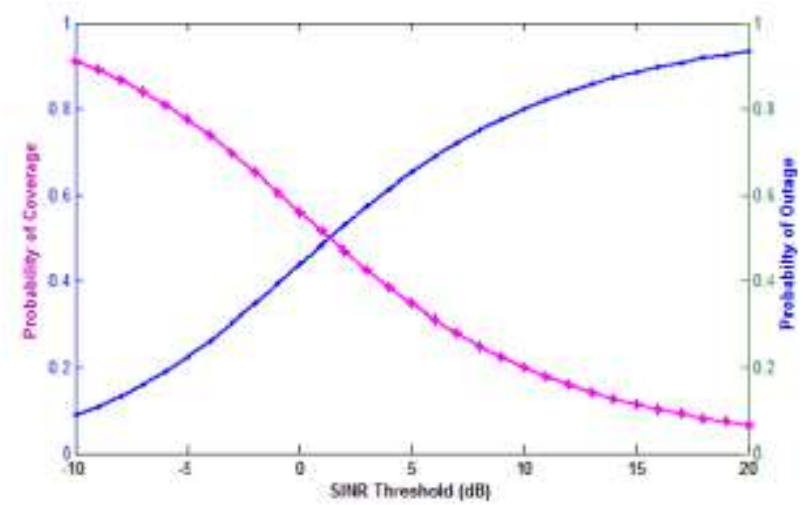

Figure 5: Coverage probability and Outage Probability with respect to SINR Threshold in $\mathrm{dB}$

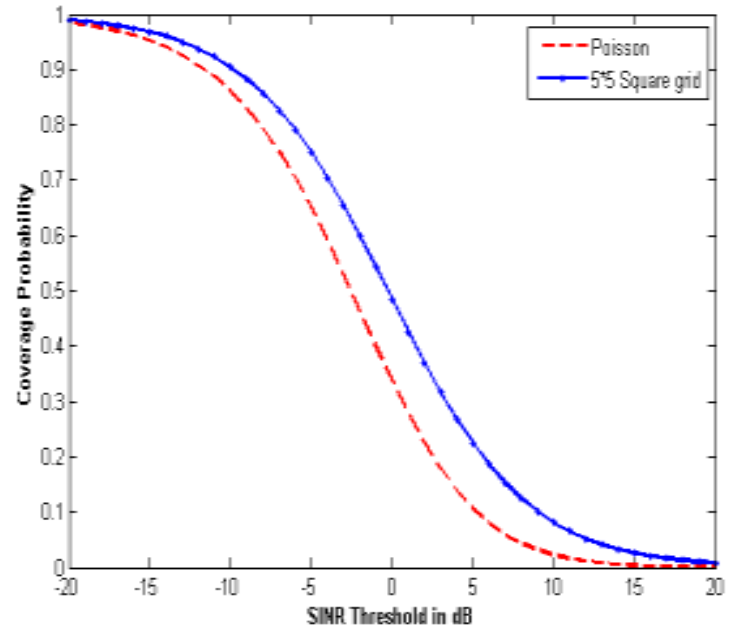

Figure 6: Coverage Probability of grid model and Random spatial model using PPP as a function of SINR Threshold in $\mathrm{dB}$

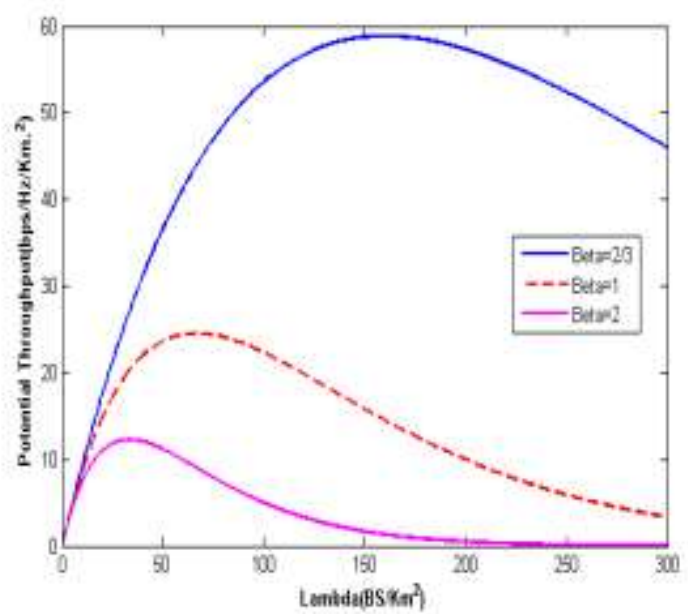

Fig. 7: Potential throughput as a function of BS Density $(\lambda)$ for different Path loss coefficient 
DOI: https://dx.doi.org/10.26808/rs.ca.i8v4.04 International Journal of Computer Application (2250-1797)

Issue 8 Volume 4, July-August 2018

Results for Coverage Probability with respect to No. of Macro BS \& Small cell Density

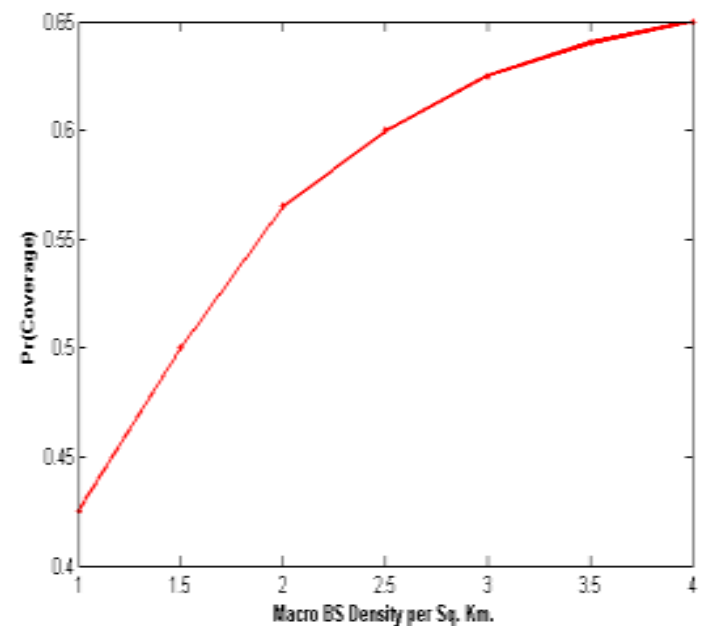

Fig.8: Coverage probability of single tier Macro BS

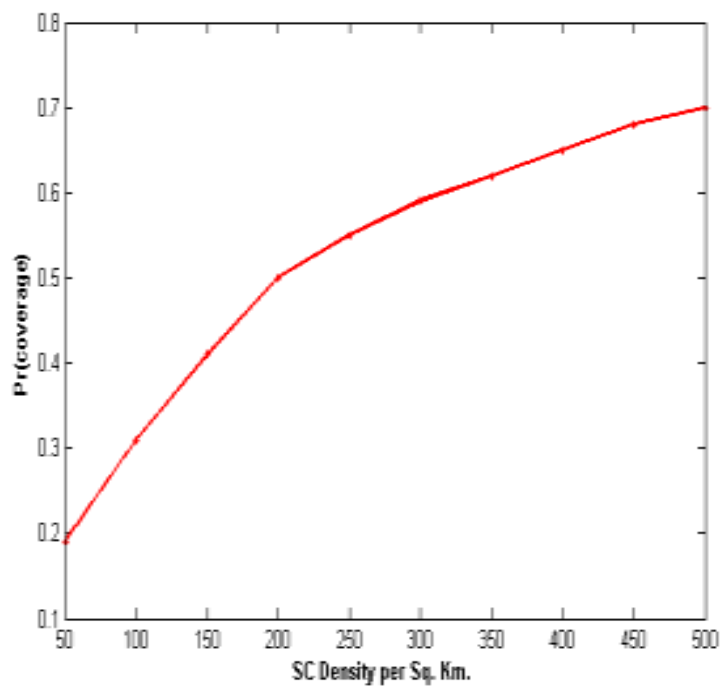

Fig.9: coverage probability of densification of small cell network

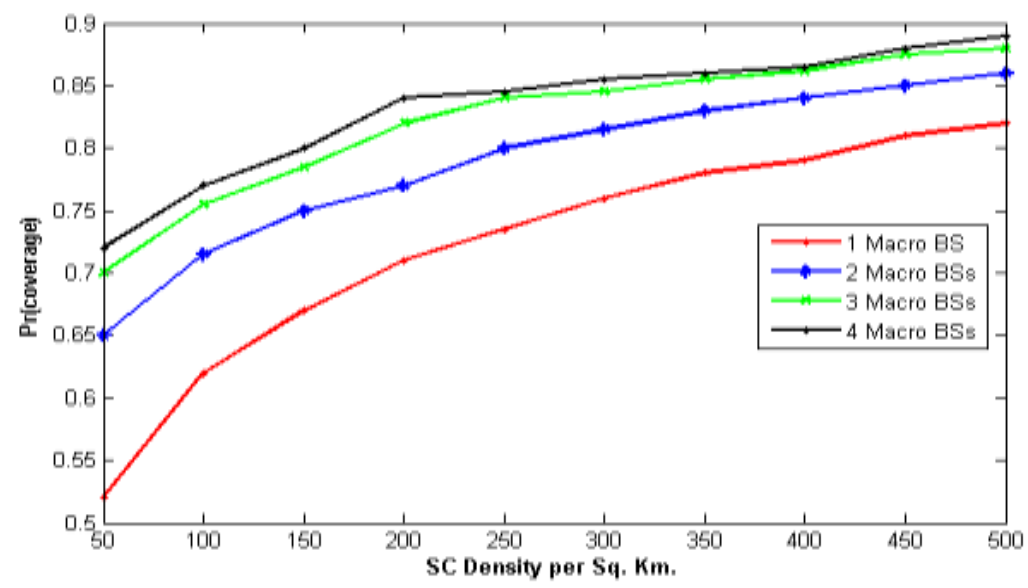

Fig.10: Coverage probability of two tier cellular network 
DOI: https://dx.doi.org/10.26808/rs.ca.i8v4.04 International Journal of Computer Application (2250-1797)

Issue 8 Volume 4, July-August 2018

\section{Result Analysis:}

The coverage probability is not affected by the no. of tiers or their relative densities and transmit powers in an interference limited network. In fact it is exactly the same as that of the single tier case. Therefore more BSs can be added in any tier without affecting the coverage and hence the net network capacity can be increased linearly with the number of BSs. The decision of a mobile user to connect to a BS depends on the received SIR from that BS and a common target SIR. It also depends upon the tier to which the BS belongs. The mobile user doesn't differentiate between the tiers when the SINR thresholds are the same. It leads to the situation where the change in received power due to change in the density or the transmit power of BSs of some tier equalized by the change in the interference power. This implies that the interference from the smaller cells such as femto, pico need not decrease the network performance in open access networks.

The grid model is frequently used as the basis of system level simulations. The actual coverage probability lies between the coverage probabilities of the PPP and the grid model. This comparison shows that the PPP assumption is as accurate as the grid model, the PPP provides a lower bound and grid model provides an upper bound to the actual coverage probability. As per fig. 5 the probability of outage can be calculated using the complement of probability of coverage.

In this paper modeling of the k-tier Heterogeneous cellular network $(\mathrm{HCN})$ and the associated analysis procedure and mathematical expressions for the coverage probability are given.

\section{Challenges:}

The challenges of the work are numerous and it includes, Physical layer technologies like multiple antennas, spread spectrum, power control and interference cancellation or interference alignment. For MAC layer, it would be scheduling, resource allocation and various forms of frequency reuse such as, fractional Frequency Reuse (FFR).

BS co-operation mechanism i.e. co-operative cluster and co-ordinaed multi point (CoMP) mechanism can be used in ultra dense cellular network for reducing no. of handovers and to reduce the congestion of the network due to unnecessary control signals for handover. Poisson cluster process can be used for clustering femto cells or pico cells in high demand areas.

\section{REFERENCES:}

1. Cisco visual networking index: Global mobile data traffic forecast update 2014-2019 white paper. Technical report, Feb. 2015

2. J.G. Andrews, S. Buzzi, Wan Choi, S.V. Hanly, A. Lozano, A.C.K. Soong, and J.C.Zhang, "What will 5G be?", IEEE Journal on Selected Areas in Communications, PP:1065 1082, June 2014

3. E. Hossain and M.Hasan, "5G cellular: key enabling technologies and research challenges" IEEE Instrumentation Measurement Magazine PP:11 21, June 2015

4. Akhil Gupta, Student Member, IEEE, Rakesh Kumar Jha, Senior Member, IEEE "A Survey of 5G Network: Architecture and Emerging Technologies" IEEE Access, Volume: 3 Pages: 1206 - 1232, Year: 2015

5. D. Lopez-Perez, I. Guvenc, G. de la Roche, M. Kountouris, T. Q. Quek, and J. Zhang, "Enhanced inter-cell interference coordination challenges in heterogeneous networks", IEEE Wireless Comm., PP: 2230, June 2011. 
DOI: https://dx.doi.org/10.26808/rs.ca.i8v4.04 International Journal of Computer Application (2250-1797)

Issue 8 Volume 4, July-August 2018

6. K. Kikuchi and H.Otsuka, "Proposal of adaptive control CRE in heterogeneous networks", In Proc. IEEE PIMRC, pages: 910914, Sydney, Australia, Sept. 2012.

7. C. S. Chen, F. Baccelli, and L. Roullet, "Joint optimization of radio resources in small and macro cell networks", In Proc. IEEE VTC 2011 Spring, pages: 15, Budapest, Hungary, May 2011

8. M. Condoluci, M. Dohler, G. Araniti, A. Molinaro, and K. Zheng, "Toward 5G densenets: architectural advances for effective machine-type communications over femtocells", IEEE Communications Magazine, pp: 134141, January 2015. ISSN 0163-6804. doi: 10.1109/MCOM.2015.7010526

9. S. Khakurel, L. Musavian, and T. Le-Ngoc, "Energy-efficient resource and power allocation for uplink multi-user OFDM systems", In Proc. IEEE PIMRC 2012, pages: 357361, Sydney, Australia, Sept. 2012

10. E. Hossain, M. Rasti, H. Tabassum, and A. Abdelnasser, "Evolution toward 5G multitier cellular wireless networks: An interference management perspective", IEEE Wireles Communications, pages:118127, June 2014. ISSN 1536-1284. doi: 10.1109/MWC.2014.6845056

11. 3GPP, TR36.814 (V11.1.0), "Further Advancements for E-UTRA Physical Layer Aspects", IEEE Wireless Communications, Mar., 2010

12. Chungang Yang et al., "Advanced Spectrum Sharing in 5G Cognitive Heterogeneous Networks", IEEE Wireless Communications, April 2016

13. N. Bhushan et al., "Network Densification: The Dominant Theme for Wireless Evolution into 5G", IEEE Commun. Mag., vol. 52, no. 2, Feb. 2014, pp: 8289

14. R. Q. Hu and Y. Qian, "An Energy Efficient and Spectrum Efficient Wireless Heterogeneous Network Framework for 5G Systems", IEEE Commun.Mag., vol. 51, no. 6, June 2014, pp: 94-101

15. J. Mitola et al, "Accelerating 5G QoE via Public-Private Spectrum Sharing", IEEE Commun.Mag., vol. 52, no. 5, May 2014, pp: 7785

16. Weisi Guo, Siyi Wangy, Xiaoli Chux, Jiming Chen, "Automated Small-Cell Deployment for Heterogeneous Cellular Networks", IEEE Commun.Mag., VOL. XX, NO. Y, 2013

17. A. Damnjanovic et al., "A survey on 3GPP heterogeneous networks", IEEE Wireless Commun.Mag., June 2011

18. A. Ganz, C. M. Krishna, D. Tang, and Z. J. Haas, "On optimal design of multitier wireless cellular systems", IEEE Wireless Commun. Mag., vol. 35, no. 2, pp: 88-93, Feb. 1997

19. V. Chandrasekhar, J. G. Andrews, T. Muharemovic, Z. Shen, and A. Gatherer, "Power control in two-tier femtocell networks", IEEE Trans. Wireless Commun., vol. 8 , no. 7, July 2009

20. V. Chandrasekhar, J. G. Andrews, "Uplink capacity and interference avoidance in two-tier femtocell networks", IEEE Trans. Wireless Commun., vol. 8, no. 7, July 2009, Available: http://arxiv.org/abs/cs.NI/0702132

21. K. Yang, Y. Wu, and H.-H. Chen, "QoS-aware routing in emerging heterogeneous wireless networks", IEEE Comm. Mag., vol. 45, no. 2, pp: 74-80, Feb. 2007

22. X. Lagrangen, "Multitier cell designs", IEEE Comm. Mag., vol. 35, no. 8, pp: 60-64, Aug. 1997

23. H. Furukawa and Y. Akaiwa, "A microcell overlaid with umbrella cell system", IEEE Veh. Technol. Conf., Stockholm, Sweden, June 1994, pp: 1455-1459

24. J. Kingman, "Poisson Processes", Oxford University Press, 1993. 
DOI: https://dx.doi.org/10.26808/rs.ca.i8v4.04 International Journal of Computer Application (2250-1797)

Issue 8 Volume 4, July-August 2018

25. M. Haenggi, J. G. Andrews, F. Baccelli, O. Dousse, and M. Franceschetti, "Stochastic geometry and random graphs for the analysis and design of wireless networks", IEEE J. Select. Areas Commun., Year: 2009, Volume: 27, Issue: 7, Pages: 1029 - 1046

26. N. Ha and L. B. Le, "Distributed Base Station Association and Power Control For Heterogeneous Cellular Networks", IEEE Trans. Vehic. Tech., vol. 63, no. 1, 2014, pp: 28296

27. R. Q. Hu and Y. Qian, "Resource Management for Heterogeneous Networks in LTE Systems", IEEE Trans. Vehic. Tech., Springer, 2014

28. F. Baccelli and S. Zuyev, "Stochastic geometry models of mobile communication networks", In Frontiers in Queueing., CRC Press, 1997, pp: 227243

29. F. Baccelli, M. Klein, M. Lebourges, and S. Zuyev, "Stochastic geometry and architecture of communication networks", J. Telecommun. Syst., vol. 7, no. 1, pp. 209227, 1997

30. He Wang; Mark C. Reed, "A novel tractable framework to analyse heterogeneous cellular networks", IEEE GLOBECOM Workshops, Year: 2011, Pages: 287 - 292

31. V. Chandrasekhar, J. G. Andrews, and A. Gatherer, Femtocell networks: a survey", IEEE Commun. Magazine, vol. 46, no. 9, pp: 59-67, Sept. 2008

32. Y. Ko and K. Moessner, "Maximum Outage Capacity in Dense Indoor Femtocell Networks with Joint Energy and Spectrum Utilization", IEEE Trans. Wireless Commun., vol. 11, no. 12, 2012, pp: 416425

33. W. Guo .et al, "Automated small-cell deployment for heterogeneous cellular networks", IEEE Commun.Mag., vol. 51, no. 5 , pp. 4653, May 2013

34. Duy Trong Ngo, Student Member, IEEE, Long Bao Le, Senior Member, IEEE, and Tho Le-Ngoc, Fellow,IEEE, "Distributed Pareto-Optimal Power Control for Utility Maximization in Femtocell Networks", IEEE TRANSACTIONS ON WIRELESS COMMUNICATIONS, VOL. 11, NO. 10, OCTOBER 2012

35. Guvenc, M.-R. Jeong, F. Watanabe, and H. Inamura, "A hybrid frequency assignment for femtocells and coverage area analysis for cochannel operation", IEEE Commun. Lett., vol. 12, no. 12, Dec. 2008

36. Jeffrey G. Andrews, Senior Member, IEEE, Franois Baccelli, and Radha Krishna Ganti, "IEEE A Tractable Approach to Coverage and Rate in Cellular Networks", IEEE TRANSACTIONS ON COMMUNICATIONS, VOL. 59, NO. 11, NOVEMBER 2011

37. D. Wyner, "Shannon-theoretic approach to a Gaussian cellular multiple-access channel", IEEE Trans. Inf. Theory, vol. 40, no. 11, pp. 17131727, Nov. 1994

38. Harpreet S. Dhillon; Radha Krishna Ganti; Jeffrey G. Andrews, "A tractable framework for coverage and outage in heterogeneous cellular networks", 2011 Information Theory and Applications Workshop, Year: 2011 ,Pages: 1 - 6

39. Vikram Chandrasekhar and Jeffrey G. Andrews, Senior Member, "IEEE Spectrum Allocation in Tiered Cellular Networks", IEEE TRANSACTIONS ON COMMUNICATIONS, VOL. 57, NO. 10

40. M.-S Alouini and A. J. Goldsmith, "Area Spectral Efficiency of Cellular Mobile Radio Systems", IEEE Trans.Vehic. Tech.", vol. 48, no. 4, July 1999, pp. 104766

41. Chungang Yang et al., "Tradeoff between Energy-Efficiency and Spectral-Efficiency by Cooperative Rate Splitting", JOURNAL OF COMMUNICATIONS AND NETWORKS", VOL. 16, NO. 2, APRIL 2014

42. L. Deng et al., "A Unified Energy Efficiency and Spectral Efficiency Trade-Off Metric in Wireless Networks", IEEE Commun. Lett.", vol. 17, no. 1, 2013, pp: 5558 
DOI: https://dx.doi.org/10.26808/rs.ca.i8v4.04 International Journal of Computer Application (2250-1797)

Issue 8 Volume 4, July-August 2018

43. X. Cong et al., "Energy- and Spectral-Efficiency Trade-Off in Downlink OFDMA Networks", IEEE Trans. Wireless Commun", vol. 10, no. 11, 2011, pp. 387486

44. Lizeth Gomez, Sithamparanathan Kandeepan, "Energy efficient handovers and performance analysis in Macro-Femto cells with radio resource constraints", 2016 International Conference on Advanced Technologies for Communications (ATC)", Year: 2016, Pages: 5459

45. Chungang Yang, Kun Guo, Min Sheng, Jiangdong Li, Jian Yue, "Energy-Efficient Capacity Offload to Smallcells with Interference Compensation", IEEE WCNC'14 Track 2 (MAC and Cross-Layer Design)",Year: 2014

46. Chungang Yang et al, "Joint Power Coordination for Spectral-and-Energy Efficiency in Heterogeneous Small Cell Networks: A Bargaining Game-Theoretic Perspective", IEEE Transactions on Wireless Communications", DOI10.1109/TWC.2015.2489215

47. Chungang Yang et al, "Interference-Aware Energy Efficiency Maximization in 5G Ultra-Dense Networks", IEEE Transactions on Communications", DOI 10.1109/TCOMM.2016.2638906

48. Bo Han, Pan Hui, Member, IEEE, V.S. Anil Kumar, Member, IEEE, Madhav V. Marathe, Senior Member, IEEE, Jianhua Shao, and Aravind Srinivasan, Fellow, IEEE, "Mobile Data Offloading through Opportunistic Communications and Social Participation", IEEE TRANSACTIONS ON MOBILE COMPUTING", DOI 10.1109/TCOMM.2016.2638906

49. C. B. Sankaran, Motorola Mobility India, "Data Offloading Techniques in 3GPP Rel10 Networks: A Tutorial", IEEE TRANSACTIONS ON MOBILE COMPUTING", DOI 10.1109/TCOMM.2016.2638906

50. Chungang Yang, Kun Guo, Min Sheng, Jiangdong Li, Jian Yue, "Energy-Efficient Capacity Offload to Smallcells with Interference Compensation", IEEE WCNC'2014 Track 2 (MAC and Cross-Layer Design)",

51. Shashi Ranjan, Nadeem Akhtar, Mahima Mehta, Abhay Karandikar, "User-Based Integrated Offloading Approach for 3GPP LTE-WLAN Network", IEEE WCNC'2014" Track 2 (MAC and Cross-LayerDesign)

52. ZHONGSHAN ZHANG , XIYUAN WANG, KEPINGLONG, ATHANASIOSV. ASILAKOS, and LA-JOSHANZO, "Large scale MIMO based wireless backhaul in 5G", IEEE Wireless Communications,October 2015

53. Xiaohu Ge, Song Tu, Guoqiang Mao, Cheng-Xiang Wang, and Tao Han, "5G UltraDense Cellular Networks", IEEE Wireless Communication, February 2016

54. J. Robson, "Small Cell Backhaul Requirements", NGMN White Paper", June 2012, pp. 140

55. Jungnickel et al., "Backhaul Requirements for Inter-Site Cooperation in Heterogeneous LTE-Advanced Networks", Proc. IEEE ICC Wksps.", June 2013, pp. 90510.

56. D. Bojic et al., "Advanced Wireless and Optical Technologies for Small-Cell Mobile Backhaul with

57. Dynamic Software-Defined Management",IEEE Commun. Magazine", Vol. 51, no. 9, Sept. 2013, pp.8693

58. ZHENGAO, LINGLONGDAI, DEMI, ZHAOCHENGWANG, MUHAMMADALIIMRAN, AND MUHAMMAD ZEESHAN SHAKIR, "MMWave massive MIMO based wireless backhaul for the 5G ultra dense network", IEEE Wireless Communications", October 2015 
DOI: https://dx.doi.org/10.26808/rs.ca.i8v4.04 International Journal of Computer Application (2250-1797)

Issue 8 Volume 4, July-August 2018

59. C.-L. I, L. J. Greenstein, and R. D. Gitlin, "A microcell/macrocell cellular architecture for low- and high-mobility wireless users", IEEE J. Select. Areas Commun.", Aug. 1993

60. T. E. Klein and S.-J. Han, "Assignment strategies for mobile data users in hierarchical overlay networks:performance of optimal and adaptive strategies", IEEE J. Select. Areas Commun.", Vol. 22, no. 5, pp. 849-861, June 2004.

61. D. Gesbert, S. Hanly, H. Huang, S. Shamai, O. Simeone, and W. Yu, "Multi-cell MIMO cooperative networks: a new look at interference", IEEE J. Select. Areas Commun.", Vol. 28, no. 9, pp. 13801408, Dec. 2010

62. N. B. Mehta, J. Wu, A. F. Molisch, and J. Zhang, "Approximating a sum of random variables with a lognormal", IEEE Trans. Wireless Commun.", vol. 6, no. 7, pp. 26902699, July 2007

63. S. Lowen and M. Teich, "Power-law shot noise", IEEE Trans. Inform. Theory", vol. 36, no. 6, pp. 1302-1318, Nov. 1990

64. S. Weber, J. G. Andrews, and N. Jindal, "The effect of fading, channel inversion, and threshold scheduling on ad hoc networks", IEEE Trans. Inform. Theory", Vol. 53, no. 11, pp. 4127-4149, Nov. 2007

65. K. Yeung and S. Nanda, "Channel management in microcell/macrocell cellular radio systems", IEEE Trans. Veh. Technol", Vol. 45, no. 4, pp.601-612, Nov. 1996

66. Mfon C. Uko; Sunday C. Ekpo; Ubong Ukommi; Rupak Kharel, "Shadowing effect on Macro-Femto heterogeneous network for cell-edge users", 2015 31st International Review of Progress in Applied Computational Electromagnetics (ACES)", Year: 2015, Pages: 12

67. Mfon C. Uko; Sunday C. Ekpo; Ubong Ukommi; Rupak Kharel, "Shadowing effect on Macro-Femto heterogeneous network for cell-edge users", 2015 31st International Review of Progress in Applied Computational Electromagnetics (ACES)", Year: 2015, Pages: 12

68. S. Shamai and A. D. Wyner, "Information-theoretic considerations for symmetric, cellular, multiple-access fading channelsparts I II", IEEE Trans. Inf. Theory", vol. 43, no. 11, pp. 18771911, Nov. 1997

69. G. Zhang, K.Yang, and H.-H. Chen, "Resource Allocation for Wireless Cooperative Networks: A Unified Cooperative Bargaining Game Theoretic Framework", IEEE Wireless Commun.", vol. 19, no. 2, Apr. 2012, pp. 3843

70. Y. Yan, J. Huang, and J.Wang, "Dynamic Bargaining for Relay-Based Cooperative Spectrum Sharing", IEEE JSAC", vol. 31, no. 8, 2013, pp. 148093

71. Q. Cao et al., "Power Allocation and Pricing in Multiuser Relay Networks using Stackelberg and Bargaining Games", IEEE Trans. Vehic. Tech.", vol. 61, no. 7, 2012, pp. 317790

72. F. Pantisano et al., "Spectrum Leasing as an Incentive Towards Uplink Macrocell and Femtocell Cooperation", IEEE JSAC", vol. 30, no. 3, 2012, pp. 61730

73. O. Simeone et al., "Spectrum Leasing to Cooperating Secondary Ad Hoc Networks", IEEE JSAC", vol. 26, no. 1, 2012, pp. 20313

74. R. Southwell, J. Huang, and X. Liu, "Spectrum Mobility Games", Proc. IEEE Conf. Computer Commun.", Year 2012, pp. 3745

75. O. Simeone, O. Somekh, H. V. Poor, and S. Shamai, "Local base station cooperation via finite-capacity links for the uplink of linear cellular networks", IEEE Trans. Inf. Theory", vol. 55, no. 1, pp. 190204,Jan. 2009 
76. M. Matinmikko et al., "Spectrum Sharing Using Licensed Shared Access: The Concept and Its Workow for LTE-Advanced Networks", IEEE Wireless Commun.,", vol. 21, no. 2, Apr. 2014, pp. 7279

77. P. Ahokangas et al., "Simple Rules for Mobile Network Operators Strategic Choices in Future Cognitive Spectrum Sharing Networks", IEEE Wireless Commun.", vol. 20, no. 2, Apr. 2013, pp. 2026

78. Alexis Sultan; Majed ElKouki; Hossam A_; Vincent Gauthier; Michel Marot, "A dynamic femto cell architecture using TV Whitespace improving user experience of urban Crowds", International Wireless Communications and Mobile Computing Conference (IWCMC)", Year: 2015 , pages: 886891

79. T. D. Novlan, R. K. Ganti, A. Ghosh, and J. G. Andrews, "Analytical evaluation of fractional frequency reuse for OFDMA cellular networks", ArXiv e-prints", Jan. 2011. Available: http://arxiv.org/abs/1101.5130

80. Yao-Liang Chung, "Energy-Saving Transmission for Green MacrocellSmall Cell Systems: A System-Level Perspective", IEEE Systems Journal", Volume: 11, Issue: 2, June 2017, Page(s): 706 - 716

81. Fidan Mehmeti, Thrasyvoulos Spyropoulos, "Performance Analysis of Mobile Data Offloading in Heterogeneous Networks", "IEEE Transactions on Mobile Computing", Volume: 16, Issue: 2, Feb. 1 2017, Page(s): 482 - 497

82. Mahima Mehta, Nirbhay Rane, Abhay Karandikar, Muhammad Ali Imran,and Barry G. Evans, "A self-organized resource allocation scheme for heterogeneous macrofemto networks", WIRELESS COMMUNICATIONS AND MOBILE COMPUTING Wirel. Commun. Mob. Comput. (2014)", DOI: 10.1002/wcm. 2518

83. Florian Letourneux, Sylvain Guivarch, Yves Lostanlen, "Propagation Models For Heterogeneous Networks" 7th European Conference on Antennas and Propagation"

84. A book on Game Theory in Wireless and Communication Networks: Theory, Models, and Applications by, Zhu Han, Dusit Niyato, Walid Saad, Tamer Baar and Are Hjrungnes, Cambridge University Press 